\title{
No association between the COMT Val158Met polymorphism and cognitive training response in healthy Shanghai community-dwelling elderly individuals
}

\author{
Wei Feng ${ }^{1 \#}, \mathrm{Ze} \mathrm{Yu}{ }^{2 \#}$, Shunying $\mathrm{Yu}^{3}$, Wenyuan $\mathrm{Wu}^{2}$, Jennifer S. Yokoyama ${ }^{4}$, Chunbo $\mathrm{Li}^{3}$ \\ ${ }^{1}$ Department of Psychological Medicine, Renji Hospital, Shanghai Jiao Tong University School of Medicine, Shanghai, China; ${ }^{2}$ Department of \\ Psychiatry, Tongji Hospital, Tongji University, Shanghai, China; ${ }^{3}$ Shanghai Key Laboratory of Psychotic Disorders, Shanghai Mental Health \\ Center, Shanghai Jiao Tong University School of Medicine, Shanghai, China; ${ }^{4}$ Memory and Aging Center, Department of Neurology, University of \\ California, San Francisco, California, USA \\ Contributions: (I) Conception and design: W Feng, Z Yu; (II) Administrative support: W Feng; (III) Provision of study materials or patients: All \\ authors; (IV) Collection and assembly of data: All authors; (V) Data analysis and interpretation: W Feng, Z Yu; (VI) Manuscript writing: All authors; \\ (VII) Final approval of manuscript: All authors. \\ "These authors contributed equally to this work. \\ Correspondence to: Wei Feng, MD, PhD. Department of Psychological Medicine, Renji Hospital, Shanghai Jiao Tong University School of Medicine, \\ 160 Pujian Road, Shanghai 200127, China. Email: ffww06@163.com.
}

Background: Cognitive training may have an effect on promoting the maintenance of cognitive functions in healthy elderly adults. The catechol-O-methyltransferase (COMT) variant Val158Met (rs4680) is a wellstudied functional variant that has been associated with differences in cognitive functions. Whether this COMT polymorphism is associated with cognitive performance after training remains unknown. We tested whether the COMT Val158Met polymorphism could influence the cognitive function over time in healthy community-dwelling elderly adults who participated in a multi-domain cognitive training intervention.

Methods: A total of 147 healthy Chinese individuals at 70 years of age and older who met the inclusion criteria were recruited from one urban community in Shanghai. One group ( $n=88$ ) accepted multi-domain cognitive training over the span of 12 weeks. The control group $(n=59)$ did not receive any intervention. Individuals underwent a multi-domain neuropsychological test four times over the course of a year (baseline, post-intervention, and 6-12 months follow-up). Composite measures of cognitive function were identified from neuropsychological test scores via factor analysis. Effects of the intervention and carrying the COMT Val158Met allele on cognitive measures were evaluated via regression models.

Results: In cross-sectional analysis there were no baseline effects of rs4680 on cognitive function $(\mathrm{P}>0.05)$ with or without adjustment for demographic covariates. There was a very subtle effect of rs 4680 on measures of processing speed at the post-intervention time point (beta $=-0.28 \pm 0.13, \mathrm{P}=0.03$ ) and at the 6 -month post-intervention time point (beta $=-0.31 \pm 0.14, \mathrm{P}=0.02)$, but not at 12 -month post-intervention $(\mathrm{P}=0.15)$. The rs4680 genotype did not exhibit any significant effect on any other cognitive measures. Furthermore, there were no significant effects of rs4680 genotype over time on cognitive measures regardless of cognitive training intervention.

Conclusions: COMT single nucleotide polymorphism rs4680 (Val158Met) does not appear to affect cognitive function over time in this cohort of individuals, nor does it interact to affect the intervention's effect on cognition over time. There are weak associations between rs4680 and processing speed but these must be interpreted with caution, as they do not appear to be consistent over time. The COMT Val158Met polymorphism does not appear to modify cognitive function following cognitive training in healthy elderly.

Keywords: Catechol-O-methyltransferase Val158Met (COMT Val158Met); cognitive training; neuropsychology; elderly 
Submitted Oct 24, 2019. Accepted for publication Apr 07, 2020.

doi: 10.21037/apm-19-416

View this article at: http://dx.doi.org/10.21037/apm-19-416

\section{Introduction}

Age-related cognitive decline can result in cognitive impairment, which diminishes the individual's quality and duration of life (1-3). Understandably, efforts to mitigate age-related cognitive decline have been explored through cognitive intervention (4-10). The Advanced Cognitive Training for Independent and Vital Elderly trial investigated the effects of single-domain cognitive training (memory, reasoning, or processing speed training) over a 10 -year span. Findings from the Advanced Cognitive Training for Independent and Vital Elderly trial suggest single-domain cognitive training affects cognitive results, leading to the possibility for this intervention to provide an opportunity to delay difficulties in daily function (11-13).

The realization of single-domain training prompted the implementation of a 10-year multi-domain cognitive intervention study to investigate the possibility of fortifying multiple cognitive functions in a single session format. This format will benefit elderly Chinese adults as they often display deficits in several cognitive domains and are also unlikely to complete multiple sessions of training (14-16). The multidomain interventions were formatted to be user friendly.

Catechol-o-methyltransferase (COMT) moderates dopamine through its contribution to catecholamine degradation. The Met and Val variants of the COMT gene lead to a functional polymorphism. The rs 4680 variant (Val158Met) exhibits decreased enzymatic activity, leading to an increase in dopamine levels in the postsynaptic neuron which are considered to increase the capacity to process information (17). On the other hand, the Met variant produces an enzyme with increased activity which leads to lower dopamine levels. Previous studies suggest that the Val COMT allele may be associated with a reduction in executive functioning in healthy subjects (18-21), schizophrenic and bipolar affective disorders.

Our previously studies reported multi-domain cognitive training reduces declines in processing speed over time. APOE 4 is associated with reductions in executive function over time, and training may attenuate $\varepsilon 4$-associated declines in processing speed. APOE 22 carriers may also benefit from training, particularly on measures of executive function and verbal memory (22). So our underlying hypothesis, was that the effect of the intervention would also differ based on COMT variant-with Val carriers showing worse cognitive outcomes and Met carriers showing better cognitive outcomes-and training effects would last for 12-month in Met carriers (22).

\section{Methods}

\section{Sample}

A total of 151 participants were recruited and screened from two neighborhoods of the Putuo District in Shanghai in 2006. Inclusion criteria were as follows: (I) at least 70 years old; (II) an ability of self-care without any physical disability or any severe physical disease; (III) no psychiatric disorders or dementia; (IV) an ability to read, write, see, and hear; (V) activities of daily living score is 19 or below; (VI) the Chinese version of the Mini-Mental State Examination score is 19 or more.

The 151 elderly individuals were divided into two groups: 90 in the intervention group and 61 in the control group. Their age ranged from 70 to 89 years old with a mean age of 74.8 [standard deviation (SD) 3.7] years old. All participants were assessed by physical and neuropsychological tests at baseline, 3 months (immediately after intervention), 9 and 15 months after enrollment. There were 147 individuals (88 in the intervention and 59 controls) agreed to have blood drawn for DNA extraction and genotyping at baseline. This study was approved by the Ethics Committee of Tongji Hospital of Tongji University [LL(H)-09-04] and all participants provided informed consent. The study was conducted in accordance with the Declaration of Helsinki (as revised in 2013). Details of the recruitment and the protocols have been published elsewhere (23).

\section{Multi-domain cognitive training}

Participants in the intervention group received multidomain cognitive training, which consisted of two onehour training sessions each week for 12 weeks (24). The 24 sessions of intervention were: story recall training (3 times), face and name training (2 times), words recall training (2 times), vocabulary learning (2 times), reasoning training (4 times), problem solving (2 times), processing speed training (map searching training, 2 times), handcrafts (2 times), physical exercise (2 times), handwriting (1 time), 
painting (2 times). Every class has 15 individuals led by one instructor. All the participants in the intervention group were encouraged to exercise in any way as much as possible when at home. No face-to-face training was provided to the control group participants.

\section{Cognitive assessment}

The Chinese version of the World Health Organization Neuropsychological Battery of Cognitive Assessment Instruments for the elderly was used for the cognitive assessment. It included nine tests of cognitive functioning: auditory verbal learning test (memory); sorting test (executive function); cancellation test (attention; processing speed); articulation and naming tests (language); minitoken test (language); motor test (memory and executive function); visual matching and reasoning test (reasoning); spatial construction test (constructional ability); trail making test (executive function) (25). The Stroop ColorWord test was also used to assess executive function (26). Neuropsychological scores were standardized (z-scores, representing number of positive or negative SDs from the mean) across all individuals for each test. Positive scores represent better performance.

\section{COMT genotyping}

The nonsynonymous variant rs4680 (Val158Met) was chosen for genotyping. Genotyping of single nucleotide polymorphisms (SNPs) was performed by the TaqMan allelic discrimination assay. The Primer Express program (Applied Biosystems) was used to design the PCR primers and the TaqMan probes. All probes were designed by the Applied Biosystems service. The standard $5 \mu \mathrm{L}$ PCR reaction was carried out using TaqMan $^{\circledR}$ Universal PCR Master Mix reagent kits under the guidelines provided, containing: $10 \mathrm{ng}$ DNA template, $2 \times$ TaqMan PCR MasterMix $2.5 \mu \mathrm{L}$, and $20 \times$ SNP assay (including primer and FAM /VIC probes) $0.25 \mu \mathrm{L}$. The program is: initial denaturation at $95{ }^{\circ} \mathrm{C}$ for $1 \mathrm{~min}$, followed by 40 cycles of denaturation at $92{ }^{\circ} \mathrm{C}$ for $15 \mathrm{~s}$, annealing at $60{ }^{\circ} \mathrm{C}$ for $60 \mathrm{~s}$. Real-time PCR was performed on an ABI Prism 7900-HT instrument with the Sequence Detection System (SDS 2.0) and the SDS Enterprise Database (Applied Biosystems).

\section{Statistical analysis}

Epidata 3.0 software was used for data entry and SPSS 17.0 software was used for preliminary data analyses. Descriptive statistics were performed using chi-squared test (categorical data), one sample $t$-test, paired $t$-test and analysis of covariance (continuous variables) depending on the type of data. Intention-to-treat analysis was used to estimate the intervention effect. Participants who participated in at least one training session and had follow-up data were included in the analysis, regardless of whether or not they completed the entire 24 sessions. Cognitive data was summarized into equation 1 (Factor 1), equation 2 (Factor 2), and equation 3 (Factor 3). The different time points of the cognitive tests are indicated by the suffix: "base" for baseline, "a" for 3 months, "b" for 9 months, and "c" for 15 months. Equation 1 data was a measurement of verbal memory, with primary loading from auditory verbal learning test. Equation 2 data was a measurement for processing speed, primary loadings from cancellation test completion time. Tasks related to set shifting were used to gather data for equation 3 , which represented executive function.

COMT SNPs were assessed for correct Hardy-Weinberg equilibrium as a quality control measure. For analysis, genotypes were recoded from "Allele 1, Both, Allele 2" to a numeric value representing the dose of minor (Allele 1, A) allele (coded as 0,1 or 2) using Stata10/MP. All COMT analyses were conducted in RStudio Version 1.0.136. Preliminary linear model analysis used the function: $\mathrm{lm}$ (formula $=\mathrm{t} \sim$ age + gender + education + COMT_dose $)$ to investigate COMT genotype effect on cognitive score. Early results revealed data from each cognitive test to be strongly associated with certain demographic variables: equation 1 has a really strong association with education, age is a strong predictor for equation 2, and gender is a strong predictor for equation 3. The observed pattern makes sense as education can influence verbal memory, age is associated with decline in processing speed, and there are studies illustrating the gender related patterns in set shifting. However, these confound variables can prevent the accurate detection of COMT genotype or intervention effect on cognitive score. Thus, age, gender, and education were included as covariates in 'adjusted' analysis.

\section{Results}

\section{Demographic and genotype distributions in intervention and control groups}

A total of 147 individuals underwent genotyping for SNP rs4680 (Val158Met). All genotypes were in Hardy-Weinberg 
Table 1 Demographic characteristics

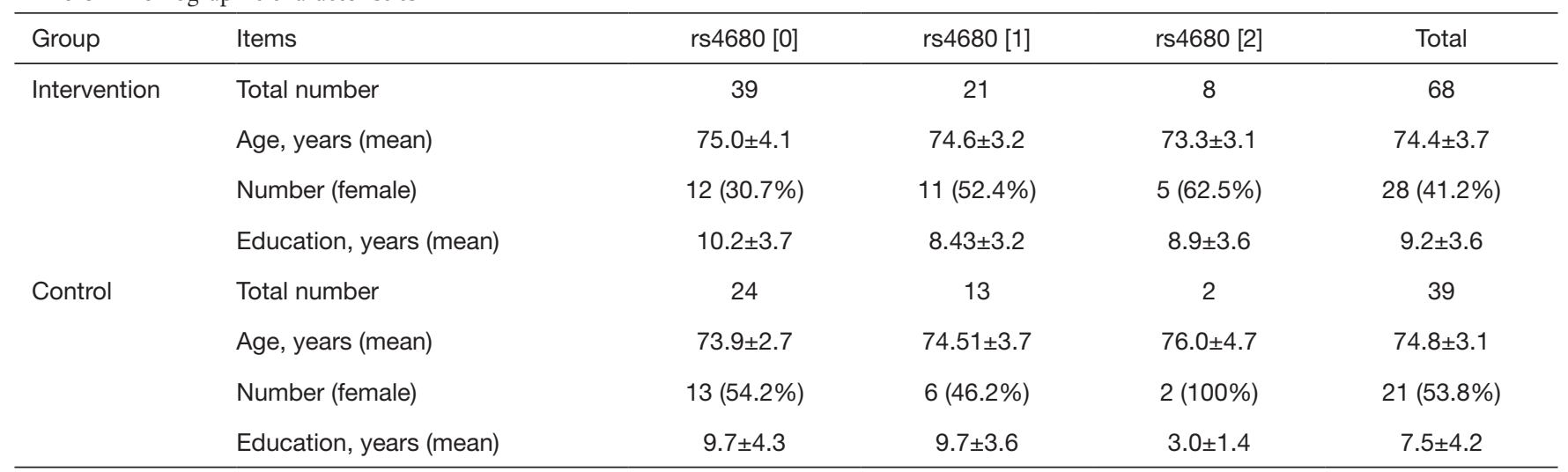

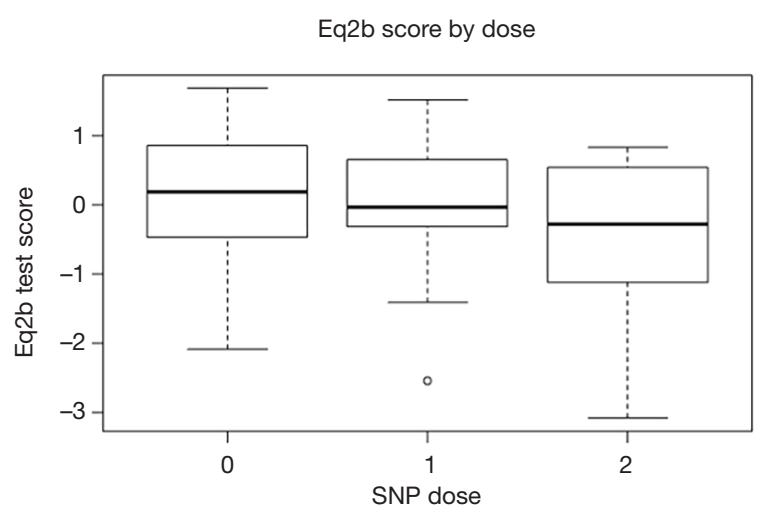

Figure 1 Box plot of factor 2 scores by dose of minor (A) allele of rs4680 at the 15 -month time point suggests that individuals exhibit a reduction in processing speed for this time point in an A allele dose dependent manner. The 'eq2b' test score is an abbreviation for 'equation 2 test score at time point b' (Equation 2 being test scores from Factor 2/processing speed assessment, and b equating to 9 -month time point).

equilibrium $(\mathrm{P}=0.60)$. There were 12 individuals with 2 minor alleles, 48 individuals with 1 minor allele, and 87 individuals with 0 minor alleles.

There were no significant differences in demographic characteristics by intervention or $r 54680$ (Val158Met) carrier status for either allele group $(\mathrm{P}>0.05$, Table 1).

\section{Genotype effects over 12-month post-intervention}

Of the 147 individuals analyzed over the course of 15 months, only 107 people had complete cognitive data for all time points.

\section{Cross-sectional analysis}

There were no baseline effects of rs 4680 on the cognitive function $(\mathrm{P}>0.05)$ with or without adjustment for demographic covariates. There was a very subtle effect of rs4680 on the processing speed (equation 2/Factor 2) at the 3 -month time point (beta $=-0.28 \pm 0.13, \mathrm{P}=0.03$ ) and at the 9-month time point (beta $=-0.31 \pm 0.14, \mathrm{P}=0.02$ ), but not at the 15 -month time point $(\mathrm{P}=0.15)$. There was no effect of rs4680 on any other measure of cognitive functions at any other time point (Figure 1).

\section{Longitudinal analysis}

There were no significant effects of rs4680 over time on any of the cognitive measures. There were no significant interactions between rs4680 $\mathrm{x}$ intervention over time on any of the cognitive measures.

\section{Discussion}

To our knowledge, this is the first study to explore the interaction between COMT SNP rs4680 (Val158Met) and cognitive function as well as the intervention's effect on cognition over time in Chinese communitydwelling elderly individuals. Through this study, we hope to evaluate if the catechol-O-methyltransferaseVal158Met-polymorphism, which is well known to affect prefrontal dopaminergic metabolism, affects both cognitive function and intervention's effect on cognition over time. Although previous studies have shown training-associated improvements on reasoning, memory and processing speed over one year post-intervention in healthy older 
adults $(11,27)$, the interaction of these results with COMT Val158Met polymorphism has not yet been thoroughly explored.

In this study, COMT SNP rs4680 (Val158Met) does not appear to affect cognitive function over time in this cohort of individuals, nor does it interact to affect the intervention's effect on cognition over time. We also evaluated different domains of cognition: memory, processing speed, and executive function so that we could identify which of these cognitive domains are the most sensitive markers of training effects.

There are weak associations between rs4680 and processing speed at the 3 -month time point (beta $=-0.28 \pm 0.13, \mathrm{P}=0.03$ ) and at the 9-month time point (beta $=-0.31 \pm 0.14, \mathrm{P}=0.02$ ), but not at the 15 -month time point $(\mathrm{P}=0.15)$. We think there are two possible reasons to explain this phenomenon. For the first one, our previous study showed that that processing speed is more dependent on white matter integrity - which shows non-linear declines that plateau in middle-age exhibit additional decline later in life (22). We can't come to the conclusion only through this study that if the intervention's effect on processing speed is also non-linear changed, but further studies to reveal the specific intervening process of the intervention seems beneficial. Second, these may be false-positives as they do not appear to be consistent effects seen over time. However, it remains to be explored why false positive results appear at both the 3-month time point and the 9-month time point.

COMT vel158met is a point mutation of the fourth exon on its gene, which leads to the activity of its product COMT reduced 3-4 times through turn the amino acid from valine (Val) into methionine (Met). For Met allele carriers, their prefrontal enzyme activity has changed, thus affects prefrontal executive function (28). An earlier study on children has shown that the effects of COMT gene polymorphism on cortical language processing may become different in a narrow age window of 6-10 years (29). In our study, subjects are 70 years of age and older, COMT SNP rs4680 (Val158Met) does not appear to affect cognitive function over time in this cohort of individuals, nor does it interact to affect the intervention's effect on cognition over time. Evidence above shows that there may be a narrow age window in which cognitive function changes in older individual, and our cohort could have missed this age.

There was no effect on rs4680 on any other measure of cognitive function at any other time point except processing speed. Our previous findings suggest that processing speed may be the most sensitive target for multi-domain cognitive training. Memory and executive function are less sensitive to training interventions because they depend more on cortical gray matter regions - which show linear rates of atrophy over time-while processing speed is more tractable to training as it is more dependent on white matter integrity-which shows non-linear declines that plateau in middle-age then show additional decline later in life $(22,30)$.

Some previous researches have shown that there is a gender difference in the activity of COMT $(31,32)$. During transcription, the estrogen could regulate the COMT expression through the estrogen receptor in its promoter region (33). And the estrogen-mediated reduction in COMT mRNA was declared to be accompanied by a decrease in COMT activity (34). In general, women have 20-30\% lower COMT activity than men (35). Additionally, evidence showed that there may be higher estrogen level in the prefrontal cortex than any other cortical area. Therefore, whether sexual difference is the impact factor of our result still need a larger sample size to authenticate.

As the first report of the interactions between COMT Val158Met polymorphism and the cognitive training in a Chinese cohort, we expect this research provides useful information to other groups designing cognitive intervention studies or with access to larger cohorts thus they can further examine these conclusions and estimate them in an independent elderly population.

\section{Limitations}

Although our findings suggest age related COMT gene polymorphisms affect cognitive function, the narrow age range of the cohort limits the strength. Future studies should focus on individuals encompassing a winder age range in order to reveal a more comprehensive understanding of age-related cognitive patterns in relation to $C O M T$ gene. Second, this time we evaluated a single COMT SNP (rs4680: Val158Met). All of COMT genes, the Val158Met is a genetic variant which was thought to be closely related to language ability, perception, and processing. Meanwhile, there is other important genelocus, as rs4818, which is was found to moderate immediate memory transfer effects following single-domain training only, with G/- carriers displaying greater benefits than $\mathrm{C} / \mathrm{C}$ carriers (36). Our previous research also involved a gene- $A p o E$, non-carriers had good response to cognitive intervention (22). In following studies, assessing the effects of multiple gene polymorphism would be beneficial. 


\section{Conclusions}

COMT SNP rs4680 (Val158Met) does not appear to affect cognitive function over time in this cohort of individuals, nor does it interact to affect the intervention's effect on cognition over time. There are weak associations between rs4680 and processing speed but these must be interpreted with caution, as they do not appear to be consistent over time. The COMT Val158Met polymorphism does not appear to modify cognitive function following cognitive training in healthy elderly.

\section{Acknowledgments}

$\mathrm{Z}$ Xue and F Fang contributed to the cognitive training validity test. Other members of the research group: Mudan Wu, Liang Liu, Jingyu Shi, Jia Xu, Qingwei Li, Xu Zhang contributed to data collection, Jeffrey Shen contributed to data analysis.

Funding: This work was supported by the National Key R\&D Program of China (2018YFC1314700); the National Nature Science Foundation of China (81371505, 81200831, 30770769); the Nature Science Foundation of Shanghai (17ZR1426400); the Fundamental Research Funds for the Central Universities (22120170037); the Top Priority of Clinical Center and Key Discipline Construction in Shanghai(2017ZZ02020); the Science and Technology Commission of Shanghai Municipality (134119a2501, 13dz2260500); Shanghai Municipal Commission of Health and Family Planning (2015ZB0502). JSY was supported by the Larry L. Hillblom Foundation 2012-A-015-FEL, an NIA Diversity Supplement to P50 AG023501 (PI: BL Miller) and the AFTD Susan Marcus Memorial Fund Clinical Research Grant.

\section{Footnote}

Conflicts of Interest: All authors have completed the ICMJE uniform disclosure form (available at http://dx.doi. org/10.21037/apm-19-416). The authors have no conflicts of interest to declare.

Ethical Statement: The authors are accountable for all aspects of the work in ensuring that questions related to the accuracy or integrity of any part of the work are appropriately investigated and resolved. The study was conducted in accordance with the Declaration of Helsinki (as revised in 2013). This study was approved by the Ethics
Committee of Tongji Hospital of Tongji University [LL(H)09-04] and all participants provided informed consent.

Open Access Statement: This is an Open Access article distributed in accordance with the Creative Commons Attribution-NonCommercial-NoDerivs 4.0 International License (CC BY-NC-ND 4.0), which permits the noncommercial replication and distribution of the article with the strict proviso that no changes or edits are made and the original work is properly cited (including links to both the formal publication through the relevant DOI and the license). See: https://creativecommons.org/licenses/by-nc-nd/4.0/.

\section{References}

1. Boyle PA, Yu L, Wilson RS, et al. Cognitive decline impairs financial and health literacy among communitybased older persons without dementia. Psychol Aging 2013;28:614-24.

2. MacDonald SW, DeCarlo CA, Dixon RA. Linking biological and cognitive aging: toward improving characterizations of developmental time. J Gerontol B Psychol Sci Soc Sci 2011;66 Suppl 1:159-70.

3. Zhu X, Yin S, Lang M, et al. The more the better? A metaanalysis on effects of combined cognitive and physical intervention on cognition in healthy older adults. Ageing Res Rev 2016;31:67-79.

4. Ball KK, Ross LA, Roth DL, et al. Speed of processing training in the ACTIVE study: how much is needed and who benefits? J Aging Health 2013;25:65S-84S.

5. Burge WK, Ross LA, Amthor FR, et al. Processing speed training increases the efficiency of attentional resource allocation in young adults. Front Hum Neurosci 2013;7:684.

6. Chapman SB, Mudar RA. Enhancement of cognitive and neural functions through complex reasoning training: evidence from normal and clinical populations. Front Syst Neurosci 2014;8:69.

7. Kelly ME, Loughrey D, Lawlor BA, et al. The impact of cognitive training and mental stimulation on cognitive and everyday functioning of healthy older adults: a systematic review and meta-analysis. Ageing Res Rev 2014;15:28-43.

8. Lampit A, Ebster C, Valenzuela M. Multi-domain computerized cognitive training program improves performance of bookkeeping tasks: a matched-sampling active-controlled trial. Front Psychol 2014;5:794.

9. Rebok GW, Langbaum JB, Jones RN, et al. Memory training in the ACTIVE study: how much is needed and 
who benefits? J Aging Health 2013;25:21S-42S.

10. Reijnders J, van Heugten C, van Boxtel M. Cognitive interventions in healthy older adults and people with mild cognitive impairment: a systematic review. Ageing Res Rev 2013;12:263-75.

11. Ball K, Berch DB, Helmers KF, et al. Effects of cognitive training interventions with older adults: a randomized controlled trial. Jama 2002;288:2271-81.

12. Rebok GW, Ball K, Guey LT, et al. Ten-year effects of the advanced cognitive training for independent and vital elderly cognitive training trial on cognition and everyday functioning in older adults. J Am Geriatr Soc 2014;62:16-24.

13. Wolinsky FD, Unverzagt FW, Smith DM, et al. The ACTIVE cognitive training trial and health-related quality of life: protection that lasts for 5 years. J Gerontol A Biol Sci Med Sci 2006;61:1324-9.

14. Cheng $\mathrm{Y}, \mathrm{Wu} W$, Feng $\mathrm{W}$, et al. The effects of multidomain versus single-domain cognitive training in nondemented older people: a randomized controlled trial. BMC Med 2012;10:30.

15. Shatil E. Does combined cognitive training and physical activity training enhance cognitive abilities more than either alone? A four-condition randomized controlled trial among healthy older adults. Front Aging Neurosci 2013;5:8.

16. Verghese J, Mahoney J, Ambrose AF, et al. Effect of cognitive remediation on gait in sedentary seniors. $\mathrm{J}$ Gerontol A Biol Sci Med Sci 2010;65:1338-43.

17. Babić Leko M, Nikolac Perković M, Klepac N, et al. Relationships of Cerebrospinal Fluid Alzheimer's Disease Biomarkers and COMT, DBH, and MAOB Single Nucleotide Polymorphisms. J Alzheimers Dis 2020;73:135-45.

18. Bruder GE, Keilp JG, Xu H, et al. Catechol-Omethyltransferase (COMT) genotypes and working memory: associations with differing cognitive operations. Biol Psychiatry 2005;58:901-7.

19. de Frias CM, Annerbrink K, Westberg L, et al. Catechol O-methyltransferase Val158Met polymorphism is associated with cognitive performance in nondemented adults. J Cogn Neurosci 2005;17:1018-25.

20. Egan MF, Goldberg TE, Kolachana BS, et al. Effect of COMT Val108/158 Met genotype on frontal lobe function and risk for schizophrenia. Proc Natl Acad Sci U S A 2001;98:6917-22.

21. Malhotra AK, Kestler LJ, Mazzanti C, et al. A functional polymorphism in the COMT gene and performance on a test of prefrontal cognition. Am J Psychiatry 2002;159:652-4.

22. Feng W, Yokoyama JS, Yu S, et al. APOE Genotype Affects Cognitive Training Response in Healthy Shanghai Community-Dwelling Elderly Individuals. J Alzheimers Dis 2015;47:1035-46.

23. Feng W, Li C, Chen Y, et al. Five-year follow-up study of multi-domain cognitive training for healthy elderly community members. Shanghai Arch Psychiatry 2014;26:30-41.

24. Williams K, Herman R, Bontempo D. Reasoning Exercises in Assisted Living: a cluster randomized trial to improve reasoning and everyday problem solving. Clin Interv Aging 2014;9:981-96.

25. Xue HB, Xiao SF, Li CB, et al. The neuropsychological test battery for elderly people. Zhonghua Yi Xue Za Zhi 2005;85:2961-5.

26. Scarpina F, Tagini S. The Stroop Color and Word Test. Front Psychol 2017;8:557.

27. Greenwood PM, Lambert C, Sunderland T, et al. Effects of apolipoprotein E genotype on spatial attention, working memory, and their interaction in healthy, middle-aged adults: results From the National Institute of Mental Health's BIOCARD study. Neuropsychology 2005;19:199-211.

28. Holtzer R, Ozelius L, Xue X, et al. Differential effects of COMT on gait and executive control in aging. Neurobiol Aging 2010;31:523-31.

29. Sugiura L, Toyota T, Matsuba-Kurita H, et al. AgeDependent Effects of Catechol-O-Methyltransferase (COMT) Gene Val158Met Polymorphism on Language Function in Developing Children. Cereb Cortex 2017;27:104-16.

30. Schiepers OJ, Harris SE, Gow AJ, et al. APOE E4 status predicts age-related cognitive decline in the ninth decade: longitudinal follow-up of the Lothian Birth Cohort 1921. Mol Psychiatry 2012;17:315-24.

31. Harrison PJ, Tunbridge EM. Catechol-Omethyltransferase (COMT): a gene contributing to sex differences in brain function, and to sexual dimorphism in the predisposition to psychiatric disorders. Neuropsychopharmacology 2008;33:3037-45.

32. Hoenicka J, Garrido E, Martinez I, et al. Gender-specific COMT Val158Met polymorphism association in Spanish schizophrenic patients. Am J Med Genet B Neuropsychiatr Genet 2010;153B:79-85.

33. Wu Q, Odwin-Dacosta S, Cao S, et al. Estrogen down regulates COMT transcription via promoter DNA 
methylation in human breast cancer cells. Toxicol Appl

Pharmacol 2019;367:12-22.

34. Jiang H, Xie T, Ramsden DB, et al. Human catecholO-methyltransferase down-regulation by estradiol. Neuropharmacology 2003;45:1011-8.

35. Pertegal M, Fenoy FJ, Hernández M, et al. Fetal Val108/158Met catechol-O-methyltransferase (COMT) polymorphism and placental COMT activity are associated with the development of preeclampsia. Fertil Steril 2016;105:134-43.e1-3.

36. Jiang J, Fiocco AJ, Cao X, et al. The Moderating Role of COMT and BDNF Polymorphisms on Transfer Effects Following Multi- and Single-Domain Cognitive Training Among Community-Dwelling Shanghainese Older Adults. Front Aging Neurosci 2018;10:198.
Cite this article as: Feng W, Yu Z, Yu S, Wu W, Yokoyama JS, Li C. No association between the COMT Val158Met polymorphism and cognitive training response in healthy Shanghai community-dwelling elderly individuals. Ann Palliat Med 2020;9(4):1396-1403. doi: 10.21037/apm-19-416 